\title{
BUILDING DETECTION FROM LiDAR DATA USING ENTROPY AND THE K-MEANS CONCEPT
}

\author{
R. C. dos Santos ${ }^{1 *}$, G. G. Pessoa ${ }^{1}$, A. C. Carrilho ${ }^{1}$, M. Galo $^{2}$ \\ ${ }^{1}$ São Paulo State University - UNESP, Graduate Program in Cartographic Sciences, \\ Presidente Prudente, São Paulo, Brazil - renato_cstos@hotmail.com, guilhermegpessoa@hotmail.com, carrilho.acc@gmail.com \\ ${ }^{2}$ São Paulo State University - UNESP, Dept. of Cartography, \\ Presidente Prudente, São Paulo, Brazil - mauricio.galo@unesp.br
}

Commission III, WG III/5

KEY WORDS: Building Detection, LiDAR Data, Entropy, Unsupervised, K-means Algorithm, Region Growing.

\begin{abstract}
:
Information obtained from LiDAR data processing is considered in a variety of applications, among them urban planning. In this context, buildings play a substantial role, since a high percentage of the urban landscape is occupied by them. In the literature, many methodologies have been developed aiming at the detection of building using remote sensing data. The approaches can be developed by applying different ideas: regularity of cluster boundary, plane fitting, radiometric data and also in geometric attribute derived from LiDAR. This paper proposes a method of building detection based on the use of the entropy concept and the K-means algorithm in which the training step is dispensed with. The experiments were performed considering two LiDAR datasets with different densities $\left(12.5 \mathrm{pts} / \mathrm{m}^{2}\right.$ and $\left.4 \mathrm{pts} / \mathrm{m}^{2}\right)$. Visual and qualitative analysis enabled verification of the potential of the proposed method, which presented satisfactory results for both datasets.
\end{abstract}

\section{INTRODUCTION}

The point cloud derived from airborne LASER scanning (ALS) systems, also known as LiDAR data, can be used to obtain and maintain accurate and up-to-date cartographic products. The geographic information system (GIS) is an example as it is applied in several contexts, for instance, urban planning, telecommunications networks planning, surveillance and transportation and evaluation of damage caused by natural disasters. In urban planning, buildings play a major role, since they occupy a high percentage of the urban area. Considering these aspects, automatic and semi-automatic building extraction have been explored by many authors (Kim and Habib, 2009, Dal Poz et al., 2009, Awrangjeb, 2016, Gavankar and Ghosh, 2018, Santos et al., 2019).

An important task in this area is related to obtaining the set of points related to each building, which is usually performed by means of region growing and RANSAC. This is commonly carried out over non-ground points: buildings, vegetation, among other high objects. In the face of the variety of objects, the derived result is composed of building and non-building clusters, the last usually being formed by vegetation points. When the aim is detection, extraction and reconstruction of building, identifying which are the building clusters is essential.

The identification of cluster types can be performed by analyzing the boundary shape of each cluster, as seen in Galvanin and Dal Poz (2012). In this case, it is assumed that building boundaries have a certain regularity, being formed by right-angled corners, unlike vegetation contours. The limitation is related to boundary extraction, since this operation is influenced by point density and the thresholds considered, as mentioned in Santos et al. (2019).

Another approach consists in adjusting planar surfaces using the magnitude of normal position vector (Kim et al, 2016) or adjusting planes to the points of each cluster (Carrilho and Galo, 2018). In this case, it is assumed that the building is represented by a plane, or set of inclined planes, whereas vegetation is formed by several small planes with different orientations. Despite being robust to noise, this approach can present problems in identifying buildings composed of curved roofs.

In addition, radiometric data can be explored in the identification process (Uzar and Yastikl, 2013). However, not all LiDAR surveys are accompanied by radiometric data. Alternatively, geometric attributes calculated from LiDAR data can be applied (Ramiya et al., 2017). In this case, the final result is directly dependent on the estimated attributes and thresholds used to separate the classes.

In this work, a method for detecting building clusters based on a geometry attribute computed from LiDAR is proposed. With this in mind, a different approach for the calculation of the entropy attribute is proposed, since an average value for each generated cluster is estimated. Considering that most of the grouping corresponds to buildings or trees, it can be expected that building clusters present a low entropy, and non-building clusters a high entropy due to multiple returns in the vegetation. The identification of building clusters is automatically performed using the K-means algorithm. The main contribution of this work consists in the proposal of a simple and automatic building identification method, dispensing with the need for training data.

\section{METHOD}

Figure 1 presents a simplified flowchart of the proposed method. In general, the method is divided into three mains steps: preprocessing, region growing and cluster building detection. The LiDAR point cloud corresponds to input data, whereas the output is represented by building and non-building clusters.

\footnotetext{
* Corresponding author
} 


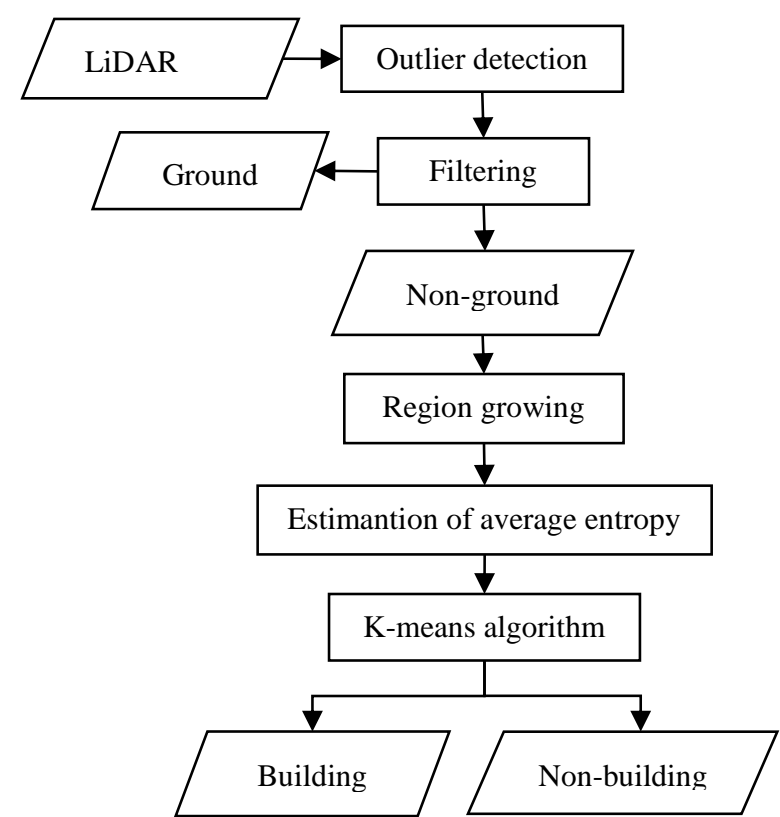

Figure 1. Flowchart of proposed method.

\subsection{Outlier Detection}

The LiDAR point cloud may contain outliers due to external factors present in the scene. The outlying points can be caused by LASER returns from objects near the scanning system, such as birds or small unmanned aircraft, and due to the multipath trajectory of the LASER pulse. According to Ben-Gal (2005), the outlying observations may lead to incorrect results. It is therefore essential to detect and eliminate them in a pre-processing step.

In this paper, the method proposed by Carrilho et al. (2018), designated as cell histogram filter ( $\mathrm{CH}$ filter), was used to perform the outlier detection. The identification process is executed by means of height histogram analysis. In this context, the outlier points are detected using a pre-established frequency threshold $\left(T_{f}\right)$.

\subsection{Filtering}

Filtering techniques are used to identify the ground and nonground points (vegetation, buildings and other high objects). This process can be designed from combination of different elements (Sithole and Vosselman, 2004): data structure, neighborhood definition criteria, measure of discontinuity, filter concept, control mechanism (single step vs. iterative), filtering nature (replacement vs. culling), and using first pulse and reflectance data.

The method proposed by Axelsson (2000) was applied to carry out the filtering process. It is an iterative approach and works in the raw point cloud. Considering the neighborhood minima criterion, a sparse TIN is obtained and, in sequence, this point set is progressively densified to the LiDAR point cloud. One point at a time for each iteration in each TIN facet is added if it meets the criteria based on the calculated threshold parameters. The parameters are the distances to the facet planes, and the angles to the nodes. The threshold parameters are estimated at each iteration considering the available data, and the iterative process ends when there is no point below the thresholds. The progressive TIN densification (Axelsson, 2000) is implemented in the lasground tool of LAStools software (http://rapidlasso.com/lastools/). In this work, this tool was applied to select the non-ground points.

\subsection{Segmentation}

According to Kwak and Habib (2014), segmentation techniques can be categorized in two approaches: region growing in the spatial domain and in the parameter (attribute) space. In the first, seed points are needed, the candidate points that satisfy a preestablished proximity criterion being attached to them. The second approach is implemented in the parameter space and consists of grouping points that have similar attributes.

In this paper, the region growing was performed in the spatial domain, being based on an approach similar to that presented by Sampath and Shan (2007). The process is executed in threedimensional space, considering the non-ground points in the original point cloud, and not the points in a grid domain as performed by Oliveira and Galo (2017). Also, two thresholds are adopted to obtain individual clusters at the region growing stage: planimetric distance $\left(T_{D x y}\right)$ and altimetric distance $\left(T_{D h}\right)$. The set of points that belong to the same grouping are stored in array $\boldsymbol{B}$, which is initially null. The steps involved in the segmentation are presented below:

Step 1: The input data are stored in array $S=\left[\begin{array}{llll}P_{o} & P_{1} & \ldots & P_{n}\end{array}\right]$.

Step 2: The first point of $\boldsymbol{S}\left(P_{o}\right)$ is adopted as seed point.

Step 3: Center a sphere of radius $R$ at the point and collect all points $\boldsymbol{G}=\left[\begin{array}{llll}r P_{1} & r P_{2} & \ldots & r P_{m}\end{array}\right]$ that fall within this sphere.

$$
R=\left[\left(L_{D x y}\right)^{2}+\left(L_{D h}\right)^{2}\right]^{1 / 2}
$$

Step 4: Verification of the closeness criteria is performed considering points located inside the sphere. The points that satisfy the criteria (Equations 1 and 2) are stored in array $\boldsymbol{A}=\left[\begin{array}{llll}c P_{1} & c P_{2} & \ldots & c P_{k}\end{array}\right]$.

$$
\begin{gathered}
{\left[\left(x_{i}-x_{c}\right)^{2}+\left(y_{i}-y_{c}\right)^{2}\right]^{1 / 2}<T_{D x y}} \\
\left|z_{i}-z_{c}\right|<T_{D h}
\end{gathered}
$$

where $x_{i}, y_{i}$ and $z i$ are coordinates of the $i$-th point inside the sphere; $x_{c}, y_{c}$ and $z_{c}$ are coordinates of the sphere center.

Step 5: Move the sphere center to $\mathrm{c} P_{1}$.

Step 6: Collect the points that satisfy the closeness criteria

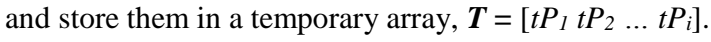

Step 7: Move the sphere center to point $\mathrm{c} P_{2}$. Append the newly collected points to array $\boldsymbol{T}$, and in this process make sure that no two points are identical.

Step 8: Continue the process until the sphere has been placed over all the points in set $\boldsymbol{A}$.

Step 9: Merge points in $\boldsymbol{A}$ and $\boldsymbol{T}$, and store them in $\boldsymbol{B}$ $(\boldsymbol{B}=\{\boldsymbol{B} \cup \boldsymbol{A} \cup \boldsymbol{T}\})$.

Step 10: Replace points in $\boldsymbol{A}$ with points in $\boldsymbol{T}$ so that the newly populated set $\boldsymbol{A}$ is equivalent to $\{\boldsymbol{T} \not \subset \boldsymbol{A}\}$

Step 11: Go back to Step 5, if at least one new point is added in $\boldsymbol{B}$. Otherwise, go to Step 12.

Step 12: When no point is added to set $\boldsymbol{B}$, array $\boldsymbol{S}$ needs to be updated. The new array $\boldsymbol{S}$ is obtained by $\boldsymbol{S}=\{\boldsymbol{S}-\boldsymbol{B}\}$. Go back to Step 2.

Step 13: Stop when there are no more points in $S$.

In order to eliminate small clusters, a criterion based on number of points is considered and segmented clusters containing less than $T_{N p}$ points are excluded. The threshold $T_{N p}$ is automatically computed considering the area of the smallest building to be represented $\left(A r_{\text {min }}\right)$ and average point density $\left(d_{\text {avg }}\right)$.

$$
T_{N p}=A r_{\text {min }} \cdot d_{a v g}
$$




\subsection{Cluster Building Detection}

In this work, the entropy concept is explored to describe the cluster characteristics. For this purpose, an average entropy value is estimated for each cluster $\left(E_{c}\right)$. The value of $E_{c}$ is obtained from the arithmetic mean of entropy values estimated for each point of grouping $\left(E_{i}\right)$, as can be seen in Equation 5. The estimated entropy value for a point $i$ is calculated from Equation 6 (Shannon, 1948, and Weinmann et al., 2015).

$$
\begin{gathered}
E_{c}=n^{-1} \sum_{E_{i}} \quad i=1,2, \ldots n \\
E_{i}=-L_{\lambda} \ln \left(L_{\lambda}\right)-P_{\lambda} \ln \left(P_{\lambda}\right)-S_{\lambda} \ln \left(S_{\lambda}\right)
\end{gathered}
$$

where:

$$
\begin{gathered}
L_{\lambda}=\left(\lambda_{1}-\lambda_{2}\right) / \lambda_{I} \\
P_{\lambda}=\left(\lambda_{2}-\lambda_{3}\right) / \lambda_{I} \\
S_{\lambda}=\lambda_{3} / \lambda_{1}
\end{gathered}
$$

correspond to linearity $\left(L_{\lambda}\right)$, planarity $\left(P_{\lambda}\right)$ and scattering $\left(S_{\lambda}\right)$, estimated from the eigenvalues $\lambda_{1}, \lambda_{2}$ and $\lambda_{3}$ considering the following ordering $\lambda_{1} \geq \lambda_{2} \geq \lambda_{3}$., as can be seen in Gross and Thoennessen (2006), Weinmann et al. (2015), and Weinmann et al. (2017).

The eigenvalues are determined from the variance-covariance matrix $\left(\boldsymbol{S}_{\boldsymbol{X}}\right)$, which is computed by means of the interest point and its neighbors (Equation 10). The neighborhood is defined by the nearest $N$ points, $N$ being obtained from the average point cloud density $\left(d_{\text {avg }}\right)$ and the area of the smallest element to be represented $\left(A r_{\min }\right)$, similar to the formulation used for estimate $T_{N P}$ (Equation 4).

$$
\begin{gathered}
\boldsymbol{S}_{\boldsymbol{X}}=N^{-1} \sum \boldsymbol{X}_{\boldsymbol{j}} \boldsymbol{X}_{\boldsymbol{j}}^{\mathrm{T}}-\boldsymbol{m}_{\boldsymbol{X}} \boldsymbol{m}_{\boldsymbol{X}}^{\mathrm{T}} \\
\boldsymbol{m}_{\boldsymbol{X}}=N^{-1} \sum \boldsymbol{X}_{\boldsymbol{j}}
\end{gathered}
$$

where:

$\boldsymbol{m}_{\boldsymbol{X}}$ - Vector of mean values;

$\boldsymbol{X}_{\boldsymbol{j}}-$ Vector of the 3D coordinates $(\mathrm{X}, \mathrm{Y}, \mathrm{Z})$, being $j=1,2, \ldots, N$.

Considering the average entropy values as input data, the next step consists of separating the clusters into two classes: buildings and non-buildings, using the K-means algorithm. This is an unsupervised classifier, the centroids being defined iteratively, as can be seen in Johnson and Wichern (2007), and Santos and Galo (2018). To perform the separation into two classes, the Euclidean distance in one-dimensional space was adopted as the similarity measure.

\section{RESULTS AND DISCUSSION}

\subsection{LiDAR Dataset}

The experiments were performed considering two LiDAR datasets. The first dataset is part of the Unesp Photogrammetric Data Set and was generated from three different flying heights over Presidente Prudente/Brazil (Tommaselli et al., 2018). This paper considers only the lowest flight. The second dataset comes from the ISPRS Test Project on Urban Classification, 3D Building Reconstruction and Semantic Labeling, which was captured over Vaihingen in Germany (Cramer, 2010). The characteristics of the LiDAR datasets are presented in Table 1.

\begin{tabular}{c|c|c}
\hline Data & P. Prudente/Brazil & Vaihingen/Germany \\
\hline $\begin{array}{c}\text { Scanning } \\
\text { system }\end{array}$ & RIEGL LMS-Q680i & $\begin{array}{c}\text { Leica Geosystems } \\
\text { ALS50 }\end{array}$ \\
\hline $\begin{array}{c}\text { Scan angle } \\
\text { Flying } \\
\text { height }\end{array}$ & $550 \mathrm{~m}$ & $45^{\circ}$ \\
\hline $\begin{array}{c}\text { Average } \\
\text { point } \\
\text { spacing }\end{array}$ & $0.3 \mathrm{~m}$ & $500 \mathrm{~m}$ \\
\hline $\begin{array}{c}\text { Average } \\
\text { point density }\end{array}$ & $12.5 \mathrm{pts} / \mathrm{m}^{2}$ & $0.7 \mathrm{~m}$ \\
\hline
\end{tabular}

Table 1. Characteristics of the LiDAR datasets used in the experiments.

\subsection{Experiments and Results}

The experiments were performed over two clippings, derived from both datasets. The first clipping (Figure 2) was selected from the Presidente Prudente dataset, and corresponds to an area with high complexity, being composed of buildings of different sizes and shapes, buildings located near each other, isolated trees, clumps of vegetation and transmission lines. A high concentration of buildings can be seen on the lower diagonal of the image in Figure 2a, with trees in the upper diagonal. The presence of trees near the buildings can also be seen, in some cases causing the occlusion of part of the building's roof. The second clipping (Figure 3) was selected from the Vaihingen dataset. It is composed of buildings of rectangular shape and isolated trees. In general, the buildings are not located too close to each other, and there are few trees in the area. In addition, there are few problems of tree occlusion in building roofs.

The thresholds considered in outlier detection $\left(T_{f}\right)$, region growing $\left(T_{D x y}, T_{D h}\right.$ and $\left.T_{N p}\right)$ and building cluster identification $(N)$ are presented in Table 2 . The value of $T_{f}$ was derived from Carrilho et al. (2018), whereas $T_{D x y}$ and $T_{D h}$ were defined by means of average point spacing $\left(T_{D x y}=2 s_{a v g}\right)$ and internal altimetric accuracy $\left(T_{D h}=\sigma_{h}\right)$, respectively. In this paper, the filtering was performed using the option "city" of the lasground tool. These values can be accessed by means of the LAStools documentation (http://www.cs.unc.edu/ isenburg/lastools/).

\begin{tabular}{c|c|c}
\hline Thresholds & P. Prudente/Brazil & Vaihingen/Germany \\
\hline $\boldsymbol{T}_{\boldsymbol{f}}$ & 5 & 5 \\
$\boldsymbol{T}_{\boldsymbol{D} x y}(\mathbf{m})$ & 0.6 & 1.4 \\
$\boldsymbol{T}_{\boldsymbol{D h}}(\mathbf{m})$ & 0.2 & 0.3 \\
$\boldsymbol{A r}_{\min }\left(\mathbf{m}^{2}\right)$ & 4 & 4 \\
$\boldsymbol{T}_{N p}=\boldsymbol{N}$ & 50 & 16 \\
\hline
\end{tabular}

Table 2. Thresholds values considered in each dataset.

The quality parameters: completeness (Comp), correctness (Corr) and $F_{\text {score }}$ (Wiedeman et al., 1998, and Sokolova et al., 2006) were computed for both areas using the proposed method (Table 3). It is important to highlight that these parameters are related only to the building class. The reference data were manually-derived on original LiDAR data using the CloudCompare software (https://www.cloudcompare.org/).

\begin{tabular}{c|c|c}
\cline { 2 - 3 } & P. Prudente/Brazil & Vaihingen/Germany \\
\hline Comp (\%) & 91.5 & 94.0 \\
Corr (\%) & 97.0 & 92.2 \\
$\boldsymbol{F}_{\text {score }}(\boldsymbol{\%})$ & 94.2 & 93.1 \\
\hline
\end{tabular}

Table 3. Quality parameters of proposed method for different datasets. 


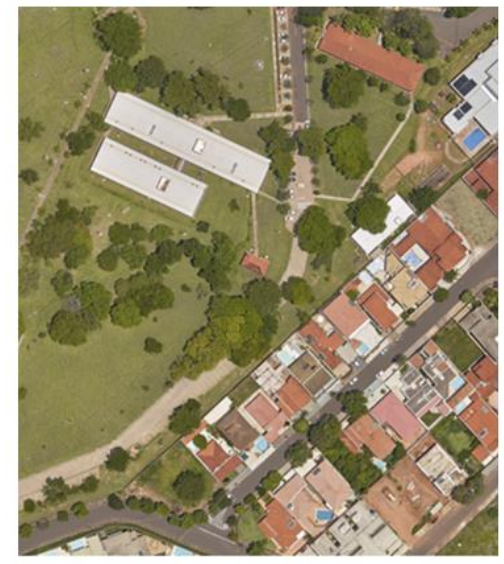

(a)

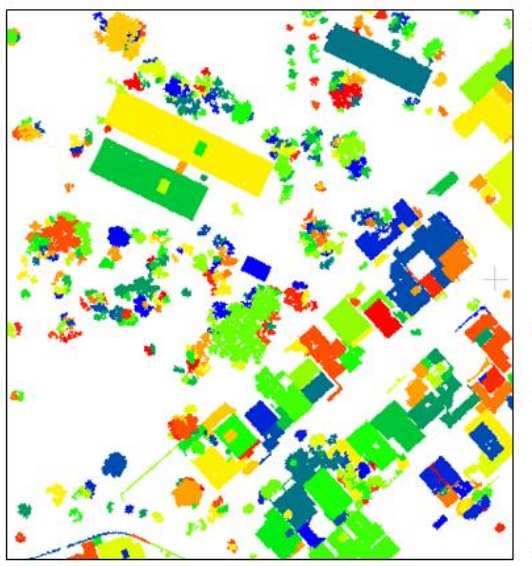

(b)

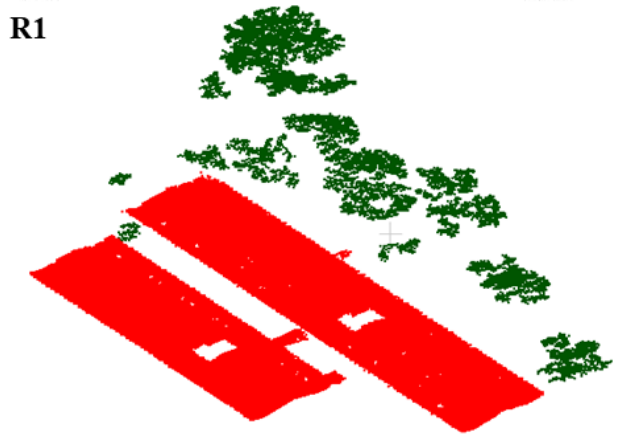

(d)

$\mathbf{R 2}$

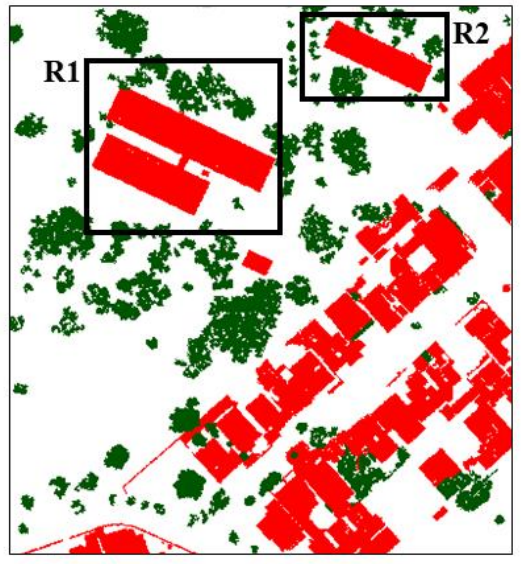

(c)

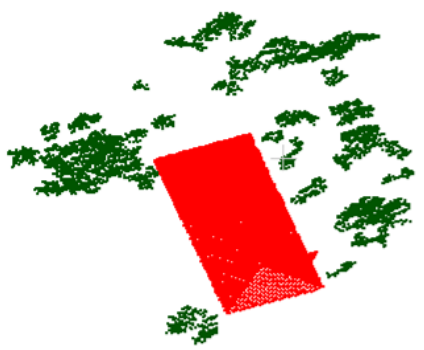

(e)

Figure 2. Presidente Prudente dataset. Aerial image (a), non-ground points after region growing process (b), and identification of building clusters using the proposed method (c).

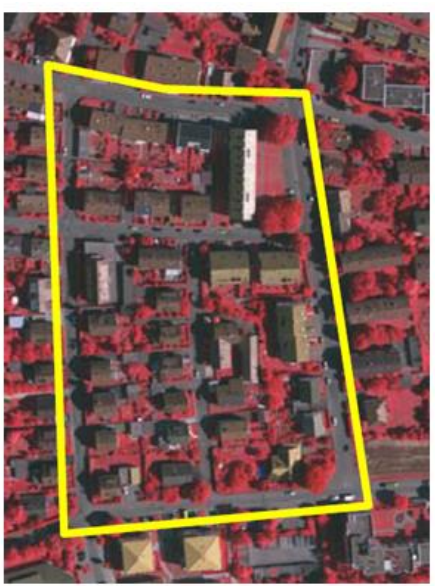

(a)

R3

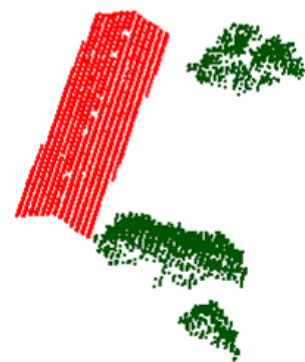

(d)

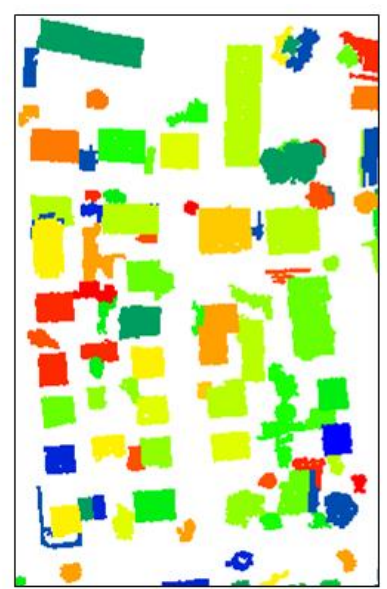

(b)

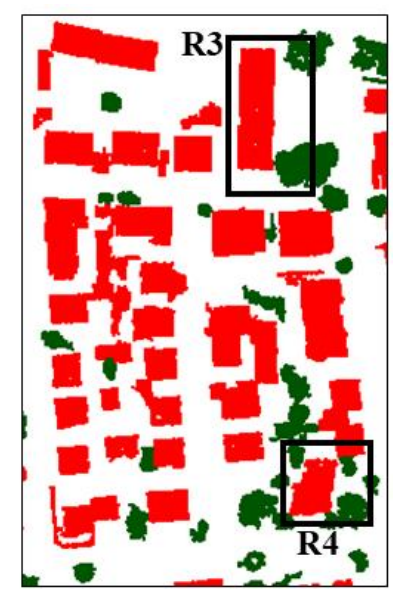

(c)

R4

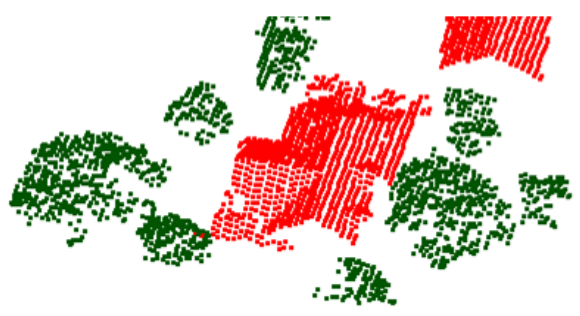

(e)

Figure 3. Vaihingen dataset. Infrared aerial image (a), non-ground points after region growing process (b), and identification of building clusters using the proposed method (c). 


\subsection{Discussion of Results}

A wide variety of objects can be seen in both areas from a visual analysis of the segmentation results. In general, the growing region process was able to identify the different clusters. Despite this, it is important to highlight that some problems may occur in the segmentation process related to the complexity of the urban scene: buildings close to each other, tree surround of the buildings, and occlusions. In this work, no process was considered to minimize these problems. The growing region results are therefore directly dependent on the threshold values adopted $\left(T_{D x y}, T_{D h}\right.$ and $\left.T_{N p}\right)$.

Considering the results derived from the building identification process (Figures 2c and 3c), it can be seen that most of the building clusters were correctly identified, even when the regions were more complex. Figures $2 \mathrm{c}$ and $3 \mathrm{c}$ highlight areas with high complexity, having vegetation surrounding the buildings. In these cases, the building clusters and non-buildings were correctly separated, including the small clusters. In addition, it can be seen that a few linear segments, usually related to power lines or walls, were identified as building clusters as highlighted in Figure 2c.

Analyzing the values of quality parameters, it can be seen that, in terms of completeness and correctness, the best results are related to dataset 2 and dataset 1 respectively. Considering the $F_{\text {score }}$ obtained by means of harmonic mean between completeness and correctness, similar results can be seen for both datasets. These results indicated that the proposed method is suitable for building cluster identification, even for areas with different characteristics.

In summary, the qualitative and quantitative analysis indicates the proposed method can be used to identify building clusters from LiDAR data automatically, presenting satisfactory results for urban areas with high levels of complexity and datasets with different specifications.

\section{CONCLUSIONS}

This paper proposes a method to identify the building clusters. The process explores the entropy concept to describe the cluster characteristics, and the K-means algorithm to separate the clusters into buildings and non-buildings. Although the proposed method is based in an unsupervised classifier, eliminating the need to run a training stage, the results are dependent on the thresholds adopted.

The experiments using two LiDAR datasets show that the proposed method generated satisfactory results even for critical regions: high concentration of buildings, tree surround the buildings, buildings with different sizes and shapes, and occlusions. However, the result derived from building identification process is directly influenced by the growing region step, characterizing a limitation of the method. As for future research, it is suggested the refinement of growing region step or the use of a more robust segmentation method, aiming to minimize problems in the mentioned critical regions in urban area.

\section{ACKNOWLEDGEMENTS}

The authors would like to thank São Paulo Research Foundation - FAPESP (grant no 2016/12167-5) and National Council for Scientific and Technological Development - CNPq (grant $\mathrm{n}^{\circ} 304189 / 2016-2$ ) for supporting this research. In addition, Sensormap Geotecnologia for providing de LiDAR data from Presidente Prudente and also the German Society for Photogrammetry, Remote Sensing and Geoinformation (DGPF) for providing the Vaihingen data set.

\section{REFERENCES}

Awrangjeb, M., 2016. Using point cloud data to identify, trace, and regularize the outlines of buildings. International Journal of Remote Sensing, 37(3), 551-579.

Axelsson, P, 2000. DEM generation from LASER scanner data using adaptive TIN models. International Archives of Photogrammetry and Remote Sensing, 33, 110-117.

Ben-Gal, I., 2005. Outlier detection, In: Maimon, O., Rockach, L. (Eds) Data mining and knowledge discovery handbook. Kluwer Academic Publishers, Netherlands, 131-146.

Carrilho, A. C., and Galo, M., 2018. Extraction of building roof planes with stratified random sample consensus. The Photogrammetric Record, 33, 363-380.

Carrilho, A. C., Galo, M., and Santos, R. C, 2018. Statistical outlier detection method for airborne lidar data. International Archives of the Photogrammetry, Remote Sensing \& Spatial Information Sciences, 42(1), 87-92. doi.org/10.5194/isprsarchives-XLII-1-87-2018.

Gavankar, N. L., and Ghosh, S. K, 2018. Automatic building footprint extraction from high-resolution satellite image using mathematical morphology. European Journal of Remote Sensing, 52(1), 182-193.

Cramer, M., 2010. The DGPF test on digital aerial camera evaluation - overview and test design. Photogrammetrie Fernerkundung - Geoinformation, 73-82. doi.org/10.1127/1432$8364 / 2010 / 0041$

Dal Poz, A., Habib, A. F., Marcato, V. J., and Correia, L. S., 2009. Uso de dados fotogramétricos no refinamento geométrico de contornos de telhados de edifícios extraídos de dados LASER. Boletim de Ciências Geodésicas, 15(3), 594-614.

Galvanin, E. A. S., and Dal Poz, A. P., 2012. Extraction of building roof contours from LiDAR data using a MarkovRandom-Field-Based approach. IEEE Transactions on Geoscience and Remote Sensing, 50(3), 981-987.

Gross, H., and Thoennessen, U., 2006. Extraction of lines from laser point clouds. International Archives of the Photogrammetry, Remote Sensing \& Spatial Information Sciences, 36, 86-91.

Johnson, R. A., and Wichern, D. W., 2007. Applied multivariate statistical analysis. Upper Saddle River, NJ: Pearson Prentice Hall.

Kim, C., and Habib, A., 2009. Object-based integration of photogrammetric and LiDAR data for automated generation of complex polyhedral building models. Sensors, 9, 5679-5701.

Kim, C., Habib, A., Pyeon, M., Kwon, G-R., Jung, J., and Heo, J., 2016. Segmentation of planar surfaces from laser scanning data using the magnitude of normal position vector for adaptative neighbourhoods. Sensors, 16, 140. 
Kwak, E., and Habib, A, 2014. Automatic representation and reconstruction of DBM from LiDAR data using Recursive Minimum Bounding Rectangle. ISPRS Journal of Photogrammetry and Remote Sensing, 93, 171-191. doi.org/10.1016/j.isprsjprs.2013.10.003

Oliveira, R. A. R. de, and Galo, M., 2017. Classificação de feições na superfície a partir de dados LiDAR e medidas de entropia e desvio padrão das altitudes. In: Annal of the XVIII Simpósio Brasileiro de Sensoriamento Remoto (SBSR), INPE, Santos, 2892-2899.

Ramiya, A. M., Nidamanuri, R. R., and Krishnan, R., 2017. Segmentation based building detection approach from LiDAR point cloud. The Egyptian Journal of Remote Sensing and Space Sciences, 20, 71-77.

Sampath, A., and Shan, J, 2007. Building boundary tracing and regularization from airborne LiDAR point clouds. Photogrammetric Engineering and Remote Sensing, 73(7), 805812.

Santos, R. C., and Galo, M., 2018. Classification of LiDAR data over building roofs using k-means and principal component analysis. Boletim de Ciências Geodésicas, 24(1), 69-84.

Santos, R. C., Galo, M., and Carrilho, A. C, 2019. Extraction of building roof boundaries from LiDAR data using an adaptive alpha shape algorithm. IEEE Geoscience and Remote Sensing Letter. doi.org/ 10.1109/LGRS.2019.2894098.

Shannon, C. E., 1948. A mathematical theory of communication. The Bell System Technical Journal, 17, 379-423.

Sithole, G., and Vosselman, G, 2004. Experimental comparison of filter algorithms for bare-Earth extraction from airborne LASER scanning point clouds. ISPRS Journal of Photogrammetry and Remote Sensing, 59, 85-101.

Sokolova, M., Japkowicz, N., and Szpakowicz, S, 2006. Beyond accuracy, f-score and roc: a family of discriminant measures for performance evaluation. In: Proceedings of the AAAI'06 workshop on Evaluation Methods for Machine Learning, 24-29.

Tommaselli, A. M. G., Galo, M., Reis, T. T., Ruy, R. S., Moraes, M. V. A., and Matricardi, W. V., 2018. Development and assessment of a data set containing frames images and dense airborne LASER scanning point clouds. IEEE Geoscience and Remote Sensing Letters, 15(2), 192-196. doi.org/10.1109/LGRS.2017.2779559

Uzar, M., and Yastikli, N., 2013. Automatic building extraction using LiDAR and aerial photographs. Boletim de Ciências Geodésicas, 19(2), 153-171.

Wiedemann, C., Heipke, C., Mayer, H., and Jamet, O., 1998. Empirical Evaluation of Automatically Extracted Road Axes. In: Bowyer, K. W; Phillips, P. J. (Eds.) Empirical evaluation techniques in computer vision. Los Alamitos, IEEE Computer Society Press, 172-187.

Weinmann, M., Jutzi, B., Hinz, S., and Mallet, C., 2015. Semantic point cloud interpretation based on optimal neighborhoods, relevant features and efficient classifiers. ISPRS Journal of Photogrammetry and Remote Sensing, 105, 286-304.
Weinmann, M., Muller, M. S., Hillemann, M., Reydel, M., Hinz, B., and Jutzi, B., 2017. Point cloud analysis for UAV-borne laser scanning with horizontally and vertically oriented line scanners - concept and first results. International Archives of the Photogrammetry, Remote Sensing \& Spatial Information Sciences, 42(2), 399-406. 